\title{
QT interval and dispersion in primary autonomic failure
}

\author{
Simon S S Lo, Christopher J Mathias, Martin St John Sutton
}

\begin{abstract}
Objective-To investigate the role of the autonomic nervous system in determining QT interval and dispersion.

Patients and methods-32 patients with chronic primary (idiopathic) autonomic failure (19 men, mean age 60 years) and 21 normal controls (11 men, mean age 59) without symptoms of ischaemic heart disease were studied retrospectively. Autonomic failure was diagnosed by a combination of symptomatic postural hypotension, subnormal plasma noradrenaline response to head-up tilt, and abnormal cardiovascular responses to standing, Valsalva manoeuvre, mental stress, cutaneous cold, isometric exercise, and deep breathing. QT intervals were measured from surface electrocardiograms and QT dispersion was defined as maximum QT - minimum QT occurring in any of the 12 leads.
\end{abstract}

Results-Mean heart rate (RR intervals) was similar in patients with autonomic failure and controls (S2 lead: 865 (132) $v$ 857 (108) ms, $P=$ NS; V2 lead: 865 (130) $v$ 868 (113) $\mathrm{ms}, P=\mathrm{NS}$ ). QT intervals measured from electrocardiogram leads S2 and V2 were significantly longer in patients than in controls (401 (40) $v 376$ (16) $\mathrm{ms}, P<0.01$; and 403 (41) $v 381$ (20) ms, $P<0.05$ respectively). The mean maximum QT interval in any lead, which is the best estimate of the maximum duration of electrical systole, was significantly longer in the patients than in controls (417 (48) $v 388$ (23) $\mathrm{ms}, P<0.005)$. Linear regression analysis of $Q T$ and $R R$ intervals for both groups showed a significant difference between the slopes of the two regression lines $(F=8.4, P<0.001)$. However, QT dispersions were similar between patients and controls.

Conclusions-Patients with primary autonomic failure have prolongation of QT intervals, indicating that the autonomic nervous system is an important determinant of QT interval. However, QT dispersion does not seem to be affected by chronic primary autonomic denervation.

(Heart 1996;75:498-501)

Keywords: QT interval and dispersion; primary autonomic failure; electrocardiography; autonomic nervous system
The QT interval is the electrocardiographic description of ventricular depolarisation and repolarisation, which when abnormal may identify patients at risk of developing ventricular fibrillation and sudden cardiac death. ${ }^{12}$ The autonomic nervous system is believed to be an important element in the genesis of malignant ventricular arrhythmias and sudden death. ${ }^{3}$ Changes in the autonomic neural tone may affect the QT interval by affecting depolarisation and repolarisation kinetics of myocardial cells through neural or receptor mediated mechanisms, or both. ${ }^{45}$ There is increasingly convincing evidence that dispersion of ventricular refractoriness is associated with ventricular instability and ventricular arrhythmias. ${ }^{67}$ Dispersion of the QT interval on the surface electrocardiogram may provide a non-invasive measure of dispersion of repolarisation. ${ }^{89}$ The influence of the autonomic nervous system on the QT interval is currently incompletely understood, partly because previous studies have investigated patients with secondary autonomic neuropathy in whom the primary disorder may have also affected the cardiovascular system. The most commonly studied group of patients are diabetics with autonomic neuropathy, ${ }^{10-12}$ in whom diabetic cardiomyopathy may occur and coronary artery disease is common. Normal individuals in whom pharmacological agents have been administered to block partially, or completely, the adrenergic receptors to mimic autonomic failure have also been studied. ${ }^{613}$ To overcome these difficulties and gain further insight into the role of the autonomic nervous system, a unique group of patients with chronic primary autonomic failure, who had no cardiac disease and were a physiological model of autonomic denervation, was studied, with the specific aims of evaluating the effects of autonomic failure on QT interval and dispersion.

\section{Patients and methods}

\section{STUDY POPULATION}

Patients with chronic primary autonomic failure from the Autonomic Unit, National Hospital for Nervous Diseases and the Neurovascular Medicine Unit, St Mary's Hospital, London were studied. None had evidence of diabetes mellitus, chronic liver disease, or amyloidosis. Apart from autonomic failure, they had either no neurological deficit (pure autonomic failure), or additional parkinsonian, cerebellar/pyramidal, or a combination of these neurological deficits, as part of the 
syndrome of Shy-Drager (often used synonymously with multiple system atrophy). ${ }^{14}$ The study population consisted of patients who had a 12 lead electrocardiogram recorded at the time that autonomic failure was diagnosed by a series of physiological and biochemical tests. ${ }^{15}$ All patients had severe symptomatic postural hypotension $(>30 \mathrm{~mm} \mathrm{Hg}$ decrease in systolic blood pressure). Sympathetic failure was indicated by the combination of a lack of pressor response to cutaneous cold, isometric exercise and mental arithmatic, an abnormal Valsalva response, and an absent or minimal response in plasma noradrenaline levels to head-up tilt. In addition, all patients had either blunted or no heart rate response to deep breathing and hyperventilation, indicating cardiac parasympathetic failure. Patients were excluded if they had any of the following: (1) history of cardiac ischaemia, (2) history of alcohol abuse, (3) arrhythmia, bundle branch block pattern or significant $Q$ waves on electrocardiogram, (4) serum creatinine of $>150$ $\mu \mathrm{mol} / 1$, (5) biochemical evidence of chronic liver disease as judged by serum albumin $<30$ $\mathrm{g} / \mathrm{l},(6)$ medication known to affect QT interval, (7) diabetes, and (8) a family history of sudden death. Seventeen patients with pure autonomic failure and 15 patients with ShyDrager syndrome were selected for the study. They were compared with a group of normal individuals of similar age and sex, recruited from the local community. The mean age of the 21 controls $(11 \mathrm{men})$ was 59 years and that of the 32 patients with chronic primary autonomic failure $(19 \mathrm{men}) 60$ years at the time that autonomic failure was diagnosed.

QT MEASUREMENT AND ANALYSIS

QT intervals were measured in a blinded fashion from routine standard 12 lead electrocardiograms recorded at $25 \mathrm{~mm} / \mathrm{s}$ at rest. The QT and RR intervals of at least one sinus beat (range 1-3) were measured from each of the 12 leads, and the mean QT and RR intervals calculated. In addition, $Q T$ and $R R$ intervals of at least five consecutive sinus beats (range 5-7) were measured from lead $S 2$, which was the rhythm strip on the same electrocardiographic tracings. The QT and RR intervals were measured manually with calipers. QT intervals were measured from the onset of the QRS complex to the end of the $T$ wave. The end of the $T$ wave was defined as the point of return to the isoelectric line (i.e. regardless of the polarity of the $\mathrm{T}$ wave).$^{16}$ For inclusion in the study, the $T$ wave had to be distinct with a clearly defined termination. Biphasic $T$ waves were measured to the time of final return to baseline. QT intervals were not measured in leads when $U$ waves were present, which were not frequently encountered in our patients. Electrocardiographic leads S2 and V2 were used for QT interval analysis. QT dispersion was defined as the difference between the maximum and minimum QT intervals occurring in any of the 12 leads. All measurements were performed by a single observer. Repeatability of the QT measurements was tested by a second observer, who measured the same QT intervals of lead S2 in 16 randomly selected electrocardiograms.

\section{ELECTROLYTE STUDIES}

Plasma potassium and calcium concentrations were measured at the same time as when the electrocardiograms were recorded.

\section{STATISTICAL ANALYSIS}

Data are presented as means (SD), and the 95\% confidence intervals (CI) for the differences between the means have been calculated. Student's $t$ test was used for unpaired data. Logarithmic transformation was carried out for skewed data. Analysis of variance was applied to the regression slopes between QT and $R R$ intervals. Differences were considered to be significant when $\mathrm{P}<0.05$.

\section{Results}

Plasma potassium $(4 \cdot 1(0 \cdot 4)$ (range $3 \cdot 2-5 \cdot 7)$ $\mathrm{mmol} / \mathrm{l})$ and corrected calcium $(2 \cdot 31(0 \cdot 11)$ (range $2 \cdot 10-2 \cdot 52) \mathrm{mmol} / \mathrm{l}$ ) concentrations in the patients were normal at the time of the study.

\section{QT INTERVAL}

Table 1 shows the RR and QT intervals measured from leads $\mathrm{S} 2$ and V2. The RR intervals were similar between the patients and controls. QT intervals were significantly longer in the patients than in controls (table 1). The $95 \% \mathrm{CI}$ for the difference between the means for lead S2-QT were $6.0-41.9 \mathrm{~ms}(P<0.01)$ and for lead V2-QT 10.0-46.0 ms $(P<0.05)$. However, QT dispersions were similar between patients and controls $(42.0(16 \cdot 8) v$ $38.6(14.4) \mathrm{ms}, P=\mathrm{NS})$. The number of leads in which QT intervals were measured was similar in the two groups (range 9-12). The mean of the maximum QT interval in any lead, which is the best estimate of the maximum duration of electrical systole, was significantly longer in the patients than in controls (417 (48) v 388 (23) ms, $\mathrm{P}<0.005$ ) (table 2).

In the 16 randomly selected electrocardiograms (six controls), QT intervals measured

Table 1 Results of $R R$ and $Q T$ intervals

\begin{tabular}{lll}
\hline & $\begin{array}{l}\text { Controls } \\
(n=21)\end{array}$ & $\begin{array}{l}\text { Patients } \\
(n=32)\end{array}$ \\
\hline Lead S2 & & \\
RR interval (ms) & $857(108)$ & $865(132)$ \\
QT interval (ms) & $376(16)$ & $401(40) \dagger$ \\
Lead V2 & & \\
RR interval (ms) & $868(113)$ & $865(130)$ \\
QT interval (ms) & $381(20)$ & $403(41)^{\star}$ \\
\hline
\end{tabular}

Results are expressed as mean (SD)

$\star \mathrm{P}<0.05 ;+\mathrm{P}<0.01$.

Table 2 Maximum $Q T$ intervals

\begin{tabular}{lll}
\hline & $\begin{array}{l}\text { Controls } \\
(n=21)\end{array}$ & $\begin{array}{l}\text { Patients } \\
(n=32)\end{array}$ \\
\hline QT max $(\mathrm{ms})$ & & \\
Mean & $388(23)$ & $417(48)^{\star}$ \\
Range & $344-420$ & $368-584$ \\
Median & 380 & 410 \\
\hline
\end{tabular}

$\star \mathrm{P}<0.005 v$ controls. 


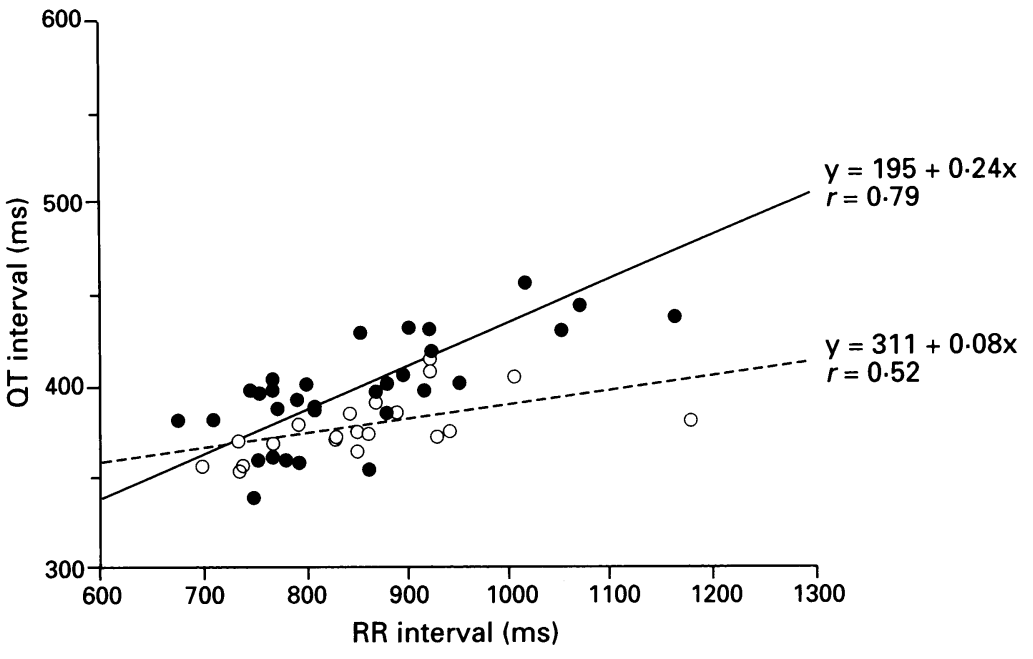

Relation between $Q T$ and $R R$ intervals derived from electrocardiogram lead S2. Patients are represented by the closed circles and continuous line, and controls by the open circles and interrupted line. and the onset of QRS is never delayed, and that the QT interval can be reliably assessed by simultaneously recording a right precordial lead and a limb lead. ${ }^{16}{ }^{17}$ We selected lead S2 as our limb lead and lead V2 as the right precordial lead.

Our study consisted of patients who when diagnosed were not taking medications that affected QT intervals. None of our patients had familial prolongation of the QT interval associated with sinus bradycardia, recurrent syncope, and sudden death from ventricular arrhythmias. ${ }^{1}$ Although we were not able to measure QT intervals in the first degree relatives of our patients, none had a family history of sudden death. Abnormalities of the QT interval have been reported in several different patient populations, including patients with diabetes, ${ }^{101118}$ myocardial ischaemia and dysfunction, ${ }^{218} 19$ alcoholism and chronic liver disease, ${ }^{2021}$ and severe hypocalcaemia or hypokalaemia. ${ }^{22}$ In our study, however, patients with symptoms or electrocardiographic evidence of myocardial ischaemia and a history of alcoholic abuse were excluded, and biochemical analysis of blood glucose, serum albumin, plasma calcium and potassium were all within normal limits. Primary autonomic neuropathy was therefore the most likely cause of the QT interval prolongation in this study.

The two limbs of the autonomic nervous system provide a normal synergistic interaction such that alteration of this balance may result in changes in QT intervals. Most human and animal studies have shown that the sympathetic system exerts a shortening effect on refractory periods and QT duration, while the parasympathetic system prolongs both these variables. ${ }^{5612} \mathrm{~A}$ sympathetic imbalance between the right and left stellate ganglia has been found in the hereditary long QT syndrome. Left cardiac sympathetic denervation was related to an improvement in frequency of syncopal attacks and patient survival. ${ }^{23}$ However, QT interval normalisation only occurred in $11.5 \%$ of the patients, suggesting that other mechanisms are involved in determining QT intervals. ${ }^{23}$ One such mechanism may be an intracardiac abnormality possibly related to potassium conductance. ${ }^{24}$ The precise mechanism causing QT interval prolongation in our patients is not clear. Sympathetic dysfunction is possibly more significant than parasympathetic dysfunction in patients with primary autonomic dysfunction, or it may be that both limbs are affected equally but the sympathetic system exerts a greater influence on the normal QT interval than the parasympathetic system.

QT interval prolongation may reflect an increased dispersion of ventricular repolarisation time and may provide a mechanism of arrhythmogenesis that involves the triggering of arrhythmias by critically timed ventricular extrasystoles. ${ }^{7-9}$ However, the normal QT dispersion in the patients indicated that the QT prolongation in primary autonomic failure is not associated with increased temporal dispersion of ventricular recovery time, but reflects a global increase in ventricular recovery time. earliest onset of the QRS complex and the deflection is frequently not simultaneous in all leads. It has been reported that the right precordial leads (V1 and V2) are the most reliable 
The abnormal QT dispersion that is well documented in patients with coronary artery disease may be linked to ischaemic related heterogeneity of repolarisation or imbalance in sympathetic activity due to regional cardiac autonomic denervation, or alterations in calcium flux. ${ }^{25}$ Certainly, the critical component is myocardial necrosis and injury, creating a dispersion of myocardial refractoriness that in conjunction with heightened sympathetic tone creates electrical instability and cardiac arrhythmias. ${ }^{25}$

This unique group of patients with primary autonomic failure provided further insights to the important influence of autonomic tone on QT interval and dispersion. Primary autonomic failure was associated with QT interval prolongation but did not increase temporal dispersion of ventricular recovery time. Autonomic neuropathy may have important prognostic implications in these patients. Some $56 \%$ of diabetic patients with autonomic neuropathy die within five years of diagnosis. ${ }^{26}$ In patients with chronic liver disease the cumulative four year mortality rate was $30 \%$ in those with autonomic neuropathy, compared with $6 \%$ in those without autonomic neuropathy. ${ }^{27}$ The six year survival rate after primary autonomic failure related to multiple system atrophy was diagnosed was $54 \%{ }^{28}$ The exact explanation for the increased death rate is unknown but sudden death due to sleep apnoea and cardiorespiratory arrest was reported in many cases. ${ }^{26-28} \mathrm{We}$ have not assessed the complex interrelation between QT interval, ventricular arrhythmias, and sudden death, although the presence of QT interval prolongation in these patients may increase their vulnerability to arrhythmia.

We thank Dr R Sutton for his expert advice and Mrs $\mathrm{K}$ Bleasdale-Barr for her technical help. CJM is supported by th Wellcome Trust and Brain Research Trust.

1 Schwartz PJ, Periti M, Malliani A. The long QT syndrome Am Heart f 1975;89:378-90.

2 Schwartz PJ, Wolf S. QT interval prolongation as predictor of sudden death in patients with myocardial infarction. Circulation 1978;57:1074-7.

3 Verrier RL, Hagestad EL. Role of the autonomic nervous system in sudden death. In: Josephson ME, ed. Sudden cardiac death. Philadelphia: David, 1985:41-63.

4 Cappato R, Alboni P, Pedroni P, Gilli G, Antonioli G Sympathetic and vagal influences on rate-dependent changes of QT interval in healthy subjects. Am $\mathcal{F}$ Cardiol 1991;68:1188-93.
5 Browne KF, Zipes DP, Heger J, Pristowsky EN. Influence of the autonomic nervous system on the QT interval. $\mathrm{Am}$ 7 Cardiol 1982;50:1099-103.

6 Ahnve S, Vallin H. Influence of heart rate and inhibition of autonomic tone on the QT interval. Circulation 1982;65: 435-9.

7 Kuo CS, Munakata K, Reddy CP, Surawitz B. Characteristics and possible mechanisms of ventricular arrhythmia dependent on the dispersion of action potential durations. Circulation 1983;67:1356-7.

8 Ambroggi LD, Negroni MS, Monza E, Bertoni T, Schwartz PJ. Dispersion of ventricular repolarisation in the long QT syndrome. Am f Cardiol 1991;68:614-20.

9 Day CP, McComb JM, Campbell RWF. QT interval dispersion: an indication of arrhythmia risk in patients with long QT intervals. Br Heart ₹ 1990;63:342-4.

10 Ewing DJ, Neilson JMM. QT interval length and diabetic autonomic neuropathy. Diabetic Med 1990;7:23-6.

11 Bellavere F, Ferri M, Guarini L, Bax G, Piccoli A, Cardone $\mathrm{C}$, et al. Prolonged QT period in diabetic autonomic neuropathy: a possible role in sudden cardiac death? $\mathrm{Br}$ Heart F 1988;59:379-83.

12 Bexton RS, Vallin HO, Camm AJ. Diurnal variation of the QT interval-influence of the autonomic nervous system. Br Heart $\mathcal{F}$ 1986;55:253-8.

13 Tonkin AM, Tornos P, Heddle WF, Rapp H. Autonomic effects on the human cardiac conduction system: evalua-
tion by intracardiac electrocardiography and protion by intracardiac electrocardiography and pro-
grammed stimulation techniques. Br Heart $\mathcal{f} 1980 ; 44$ : grammed

14 Mathias CJ, Gunnar M. Autonomic dysfunction, hypotension and shock. In: Willerson JT, Cohn JW, eds. Cardiovascular medicine. New York: Churchill-Livingstone, vascular medicine.

15 Mathias CJ, Bannister R. Investigation of autonomic disorders. In: Bannister R, Mathias CJ, eds. Autonomic failure. $A$ textbook of clinical disorders of the autonomic nervous system. Oxford: Oxford University Press, 1992:255-90.

16 Cowan JC, Yusoff K, Moore M, Amos PA, Gold AE, Bourke JP, et al. Importance of lead selection in QT interval measurement. Am $\mathcal{F}$ Cardiol 1988;61:83-7.

17 Wanderman KL, Loutaty G, Ovsyshcher I, Cantor A, Gussarsky Y, Gueron M. Choice of electrocardiogram leads for recording the earliest QRS onset in noninvasive measurements. Circulation 1981;63:933-7.

18 Lo SSS, St John Sutton M, Leslie RDG. Information on type 1 diabetes mellitus and QT interval from identical twins. Am $\mathcal{F}$ Cardiol 1993;72:305-9.

19 Kramer B, Brill M, Bruhn A, Kubler W. Relationship between the degree of coronary artery disease and left ventricular function and the duration of $Q T$ interval in ECG. Eur Heart $₹$ 1986;7:14-24.

20 Kempler P, Varadi A, Szalay F. Autonomic neuropathy and prolongation of the QT interval in liver disease. Lancet 1992;340:318.

21 Thuluvath PJ, Triger DR. Autonomic neuropathy and chronic liver disease. $Q f$ Med 1989;72:737-47.

22 Surawicz B. The relationship between electrocardiogram and electrolytes. Am Heart $\mathcal{f}$ 1967;73:814-9.

23 Schwartz PJ, Locati EH, Moss AJ, Crampton RS, Trazzi R, Ruberti U. Left cardiac sympathetic denervation in the therapy of congenital long OT syndrome-a worldwide therapy of congenital long QT syndrc

24 Moss AJ. Prolonged QT interval syndromes. $\mathcal{F} A M A$ 1986:256:2985-7.

25 Moss AJ, Schwartz PJ. Delayed repolarisation (QT or QTU prolongation) and malignant ventricular arrhythmias. Mod Concepts Cardiovasc Dis 1982;51:85-90.

26 Ewing DJ, Campbell IV, Clarke BF. The natural history of diabetic autonomic neuropathy. $Q \mathcal{F}$ Med 1980;49: 95-108.

27 Hendrickse MT, Thuluvath PJ, Triger DR. Natural history of autonomic neuropathy in chronic liver disease. Lancet 1992;339:1462-4.

28 Saito Y, Matsuoka Y, Takahasi A, Ohno Y. Survival of patients with multiple system atrophy. Internal Med 1994; 33:321-5. 